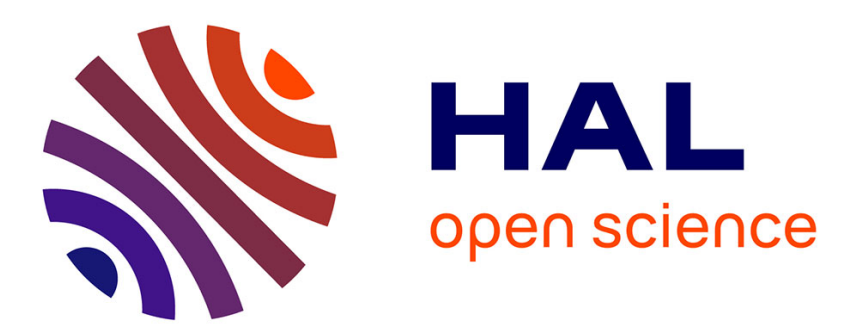

\title{
The Transferable Belief Model for reliability analysis of systems with data uncertainties and failure dependencies
}

\author{
Mohamed Sallak, Walter Schön, Felipe Aguirre
}

\section{To cite this version:}

Mohamed Sallak, Walter Schön, Felipe Aguirre. The Transferable Belief Model for reliability analysis of systems with data uncertainties and failure dependencies. Proceedings of the Institution of Mechanical Engineers, Part O: Journal of Risk and Reliability, 2010, 224 (4 / 2010), pp.266-278. hal-00522460v2

\section{HAL Id: hal-00522460 \\ https://hal.science/hal-00522460v2}

Submitted on 12 Nov 2010

HAL is a multi-disciplinary open access archive for the deposit and dissemination of scientific research documents, whether they are published or not. The documents may come from teaching and research institutions in France or abroad, or from public or private research centers.
L'archive ouverte pluridisciplinaire HAL, est destinée au dépôt et à la diffusion de documents scientifiques de niveau recherche, publiés ou non, émanant des établissements d'enseignement et de recherche français ou étrangers, des laboratoires publics ou privés. 


\title{
Transferable Belief Model for incorporating failure dependencies in reliability analysis under data uncertainties
}

\author{
Felipe Aguirre, Mohamed Sallak and Walter Schön \\ Compiegne University of Technology, UMR CNRS 6599 \\ Heudiasyc BP 20529, 60205 Compiegne cedex, France \\ Email: (felipe.aguirre-martinez,mohamed.sallak,walter.schon)@utc.fr
}

\begin{abstract}
Dealing with uncertainty introduces an increased level of complexity to reliability analysis problems. The uncertainties associated to reliability studies usually arise from the difficulty to account for incomplete or imprecise reliability data and complex failure dependencies. This paper introduces the Transferable Belief Model (TBM) to the reliability analysis so that epistemic uncertainties can be taken into account as well as aleatory uncertainties. Two approaches are used to represent failure dependencies of components: an implicit and an explicit approach. The TBM model is then compared to an intervalprobability model by highlighting the different characteristics of the results obtained.
\end{abstract}

Keywords: Transferable Belief Model (TBM), DempsterShafer (D-S) theory, reliability analysis, epistemic uncertainty, failures dependencies, interval-probability.

\section{INTRODUCTION}

Uncertainties are one of the most challenging problems in reliability studies of complex systems [1]-[3]. They are present in any reliability evaluation due to randomness in the failure phenomena and difficulty to obtain failure data of components with scarce failures. Uncertainties have been classified into two subtypes: aleatory uncertainty and epistemic uncertainty. Aleatory uncertainty is also called irreducible and inherent uncertainty. It is the inherent variation associated with the physical system or the environment under consideration [4]. It represents, for example, the inherent variability of failures and repair times of equipment. Epistemic uncertainty is subjective and reducible because it arises from lack of knowledge or data. It represents uncertainty of the outcome due to lack of knowledge or information in any phase or activity of the modeling process [4]. That's why it is important that aleatory and epistemic uncertainties are properly accounted for in reliability studies.

Classical probability theory is adapted only for aleatory uncertainty [5]. Epistemic uncertainty can be handled by possibility theory, Dempster-Shafer (D-S) theory, interval analysis, and imprecise probabilities [6]. The possibility theory is usually employed to quantify only epistemic uncertainty. The D-S theory can be considered a generalization of classical probability theory and also as a generalization of possibility theory [1]. The D-S theory has several interpretations such as the Transferable Belief Model (TBM). The TBM is completely dissociated from any model based on probability functions and it separates the credal and the decision levels [7]. Hence, in this work the TBM is proposed to handle both aleatory and epistemic uncertainties in order to evaluate the system's reliability. This work only takes into account the credal level, but the decision level can also be studied in our case.

Furthermore, in many reliability studies, the failure of system's components are assumed to be independent. However, in reality, different types of dependencies can be involved, making the results of reliability evaluations wrong. Fricks and Trivedi [8] have proposed a classification of failure dependencies (Common Cause Failures (CCFs), standby dependencies, etc.). There are two principal methods to model failure dependencies in system's reliability analysis: implicit and explicit methods [9]. The implicit method corresponds to the case of the use of joint probabilities, correlations values or conditional probabilities [10]. In explicit methods, the causes of dependencies are explicitly included into the system's logic model [10] as a block in Reliability Block Diagrams (RBDs) or a basic event in Fault Trees (FTs). Here, it is proposed to use both approaches in the TBM model reliability analysis.

Section II treats the related work using the Dempster-Shafer theory in reliability analysis. Section III presents the basic notions of the TBM model. Next, the proposed TBM approach is presented in section IV. In section V, failure dependencies are treated using an implicit and explicit approach. The TBM and interval-probability models are applied and a comparison between both approaches is given. Finally, the paper finishes with some conclusions and perspectives.

\section{RELATED WORK}

The first work introducing D-S theory in reliability analysis was presented by Dempster and Kong [11]. They proposed the use of a FT as a particular case of the tree of cliques to propagate beliefs through the tree. The prior beliefs of basic events of the tree represent prior failure beliefs of components. The second work was presented by Guth in 1991 [12] and concerned FT analysis. Guth represented the belief that a basic event $A$ happens with failure probability $p$ by three valued logic (True, False and unknown) and proposed truth tables with the three valued logic in order to propagate the beliefs in FT. Chin et al. [13] proposed to use evidence theory to capture the non-specificity and conflict features in judgment 
experts. The beliefs are then propagated in a FT in order to diagnose the fault distribution of web service process. Walley [6] and Kozin et al. [14] turned out that in some applications the use of Dempster's combination rule led to incorrect results. Almond [15] developed graphical models using belief functions and applied this graphical model in FT analysis. Rakowsky et al. [16] have modeled uncertainties in Reliability-Centered Maintenance (RCM). They used belief and plausibility measures to express the uncertainties of experts in reasoning. They also use weighted recommendations during the RCM process. This approach was applied to a fire detection and extinguishing system. Pashazadeh et al. [17] proposed reliability assessment under epistemic uncertainty using D-S and vague set theories. They eliminated the gap between the representation of combined evidence and the way of representing the components reliability in the Vague Set theory. Simon et al. [18] have proposed to combine Bayesian networks and D-S theory to study the reliability of systems under imprecise reliability data. They used evidential networks and junction tree inference algorithms.

Furthermore, there is very little work treating the use of TBM theory to model failures dependencies in reliability studies. Almond [15] proposed to treat the problem of dependence between basic events by using pivotal variables and information dependence breaking theorem. Walley [6] proposed an example which indicated that D-S theory is not suited to treat dependency in the case of total ignorance of dependencies. Hence, an original TBM reliability analysis is proposed in order to take into account failures dependencies in reliability evaluations.

\section{Basic notions of Transferable Belief Model} (TBM)

The TBM was introduced by Smets and Kennes [19] as a subjectivist interpretation of D-S theory. The D-S theory, also called evidence theory, was first described by Dempster in the 1960's [20] with the study of upper and lower probabilities and extended by Shafer in 1976 [21]. The TBM represents a unique framework for representing and manipulating aleatory and epistemic uncertainties. It is based on two levels: the credal level, where available pieces of information are represented by belief functions; and the pignistic or decision level, where masses are transformed into pignistic probabilities. It was originally applied in information fusion [22], [23], pattern recognition [24], [25] and diagnostic [26]. In a finite discrete space, the TBM can be interpreted as a generalization of probability theory where probabilities can be assigned to any subsets instead of singletons only. In this section, basic notions, extended operations, and terminology of TBM are explained. For a more detailed exposition see [19]-[21].

\section{A. Frame of discernment}

The frame of discernment $\Omega$ is the definition domain of the variable of interest $\mathbf{x}$. It consists of all mutually exclusive elementary propositions. It can be viewed as the sample space in probability theory. As an example, let's consider
$\Omega=\left\{x_{1}, x_{2}\right\}$ be a frame of discernment. Then, $x_{1}$ and $x_{2}$ are elementary propositions and mutually exclusive to each other. The power set $2^{\Omega}$ is the set of all the subsets of $\Omega$ including itself, i.e.: $2^{\Omega}=\left\{\{\emptyset\},\left\{x_{1}\right\},\left\{x_{2}\right\}, \Omega\right\}$.

\section{B. Basic Probability Assignment (BPA)}

A Basic Probability Assignment (BPA) on $\Omega$, also called Basic Belief Assignment (BBA), is a function, $m^{\Omega}: 2^{\Omega} \rightarrow$ $[0,1]$, such that:

$$
\sum_{A \in 2^{\Omega}} m^{\Omega}(A)=1
$$

The number $m^{\Omega}(A)$ represents the belief value assigned to the subset $A$ of $\Omega$. The subsets $A \subset \Omega$ such that $m^{\Omega}(A)>0$ are called focal sets of $m^{\Omega}$. A BPA having a singleton $\{x\}$ $(x \in \Omega)$ as a unique focal set represents full knowledge. A BPA having only singletons as focal sets is equivalent to probabilities. A BPA having $\Omega$ as a unique focal set represents complete ignorance and is called vacuous.

\section{Belief and plausibility functions}

The belief $\mathrm{Bel}$ and plausibility $\mathrm{Pl}$ functions for a subset $A$ are defined as following:

$$
\begin{aligned}
\operatorname{Bel}(A) & =\sum_{B \subseteq A} m^{\Omega}(B) \\
\operatorname{Pl}(A) & =\sum_{B \cap A \neq \emptyset} m^{\Omega}(B) \quad \forall A \subseteq \Omega, \forall B \subseteq \Omega
\end{aligned}
$$

$\operatorname{Bel}(A)$ measures the total assignment of belief to $A$ and all its subsets. The plausibility function measures the extent to which we fail to disbelieve the hypothesis of $A$. $[\operatorname{Bel}(A), P l(A)]$ can be viewed as the confidence interval which describes the uncertainty of $\mathrm{A}$.

\section{Combination rules}

Consider two distinct pieces of evidence $m_{i}^{\Omega}$ and $m_{j}^{\Omega}$ from two different sources $i$ and $j$. In TBM, the principal combination rules are the conjunctive and disjunctive combination rules [21]. The Dempster rule of combination is defined as the conjunctive combination of two normal BPAs followed by normalization. This rule is also called the orthogonal sum of evidence. It is defined as follows:

$$
m_{i \oplus j}^{\Omega}(H)=\frac{\sum_{A \cap B=H, \forall A, B \subseteq \Omega} m_{i}^{\Omega}(A) m_{j}^{\Omega}(B)}{1-k}
$$

With: $k=\sum_{A \cap B=\emptyset, \forall A, B \subseteq \Omega} m_{i}^{\Omega}(A) m_{j}^{\Omega}(B)$

The number defined by $k$ is called the conflict factor between the two pieces of evidence $i$ and $j$.

As mentioned by some reliability researchers [6], [14], Dempster combination rule sometimes generates wrong conclusions in the case of serious conflict between evidences. In this case, it is recommended to investigate the given information or to collect more information. Several combination rules have been defined and they often differ by the way the evidence mass of an empty intersection is allocated [27], [28]. 


\section{E. Operations on Joint Spaces}

Consider a BPA $m^{\Omega_{x} \Omega_{y}}$ defined on the Cartesian product $\Omega_{x} \Omega_{y}$. The marginal BPA $m^{\Omega_{x} \Omega_{y} \downarrow \Omega_{x}}$ on $\Omega_{x}$ is defined by:

$$
\begin{gathered}
m^{\Omega_{x} \Omega_{y} \downarrow \Omega_{x}}(A)=\sum_{B \subseteq \Omega_{x} \Omega_{y} / \operatorname{Proj}\left(B \downarrow \Omega_{x}\right)=A} m^{\Omega_{x} \Omega_{y}}(B) \\
\forall A \subseteq \Omega_{x}
\end{gathered}
$$

Where $\operatorname{Proj}\left(B \downarrow \Omega_{x}\right)=\left\{x \in \Omega_{x} / \exists y \in \Omega_{y},(x, y) \in B\right\}$. The inverse operation is a particular instance of vacuous extension. Consider a BPA $m^{\Omega_{x}}$ defined on $\Omega_{x}$. Its vacuous extension on $\Omega_{x} \Omega_{y}$ is defined by:

$$
\begin{gathered}
m^{\Omega_{x} \uparrow \Omega_{x} \Omega_{y}}(B)= \begin{cases}m^{\Omega_{x}}(A) & \text { if } B=A \times \Omega_{y} \\
0 & \text { otherwise. }\end{cases} \\
\qquad A \subseteq \Omega_{x}
\end{gathered}
$$

Let $m^{\Omega_{x} \Omega_{y}}$ denote a BPA on $\Omega_{x} \Omega_{y}$ (with underlying variables $(\mathbf{x}, \mathbf{y})$ ), and $m_{y} \Omega_{x} \Omega_{y}$ the BPA on $\Omega_{x} \Omega_{y}$ with single focal set $\Omega_{x}\{y\}$. The conditional BPA of $\mathbf{x}$ given $\mathbf{y}=y$ is defined as:

$$
m^{\Omega_{x}}[y]=\left(m^{\Omega_{x} \Omega_{y}} \oplus m_{y}^{\Omega_{x} \Omega_{y}}\right)^{\downarrow \Omega_{x}}
$$

The conditioning operation for belief functions has the same meaning as in Probability Theory. However, it also admits an inverse operation called the ballooning extension. Let $m^{\Omega_{x}}[B]$ denote the conditional BPAs on $\Omega_{x}$, given $B$. The ballooning extension of $m_{x}^{\Omega}[B]$ on $\Omega_{x} \Omega_{y}$ is the least committed BPA, whose conditioning on $B$ yields $m^{\Omega_{x}}[y]$. It is obtained as:

$$
\begin{gathered}
m^{\Omega_{x}}[B]^{\Uparrow \Omega_{x} \Omega_{y}}(C)=\mathbb{1}_{C} \cdot m^{\Omega_{x}}[B](A) \\
\mathbb{1}_{C}= \begin{cases}1 & \text { if } C=(B \times A) \cup\left(B^{c} \times \Omega_{x}\right) \\
0 & \text { otherwise. }\end{cases} \\
\forall C \subseteq \Omega_{x} \Omega_{y}
\end{gathered}
$$

In order to optimize the TBM operations and saving time and space, some computation algorithms were given in [15], [29].

\section{THE PROPOSED TBM RELIABILITY ANALYSIS}

In this paper, both system and components are allowed to take only two possible states: either working $(W)$ or failed $(F)$ (Binary State assumption). Using BPAs of functioning and failure of system components, the goal is to obtain the reliability of the whole system in the case of a parallel system.

\section{A. Frame of discernment}

Due to the Binary State assumption, the frame of discernment $\Omega_{i}$ of a component $i$ is given by: $\Omega_{i}=\left\{F_{i}, W_{i}\right\} . F_{i}$ and $W_{i}$ represent respectively the failure and the working states of the component $i$. The frame of discernment of components 1,2 and $S$ are then: $\Omega_{1}=\left\{F_{1}, W_{1}\right\}, \Omega_{2}=\left\{F_{2}, W_{2}\right\}$ and $\Omega_{S}=\left\{F_{S}, W_{S}\right\}$.

\section{B. BPAs, belief and plausibility functions of system's compo-} nents

BPA structure is more natural and intuitive way to express one's degree of belief in a hypothesis where only partial evidence is available. In reliability studies, based on expert's opinion and experimental data, BPAs of components are computed directly and this computation needs some reliability expert's efforts. The BPAs assigned to system's components by expert's opinion and experimental data can be then expressed by:

$$
\begin{array}{ll}
m^{\Omega_{i}}\left(\left\{F_{i}\right\}\right) & =f_{i} \\
m^{\Omega_{i}}\left(\left\{W_{i}\right\}\right) & =w_{i} \\
m^{\Omega_{i}}\left(\left\{W_{i}, F_{i}\right\}\right) & =1-w_{i}-f_{i}
\end{array} \quad ; \quad i=1,2
$$

Using Eq. (2), belief and plausibility functions of components 1 and 2 are computed. For example, If component 1 is considered, then: $\operatorname{Bel}\left(\left\{F_{1}\right\}\right)=m^{\Omega_{1}}\left(\left\{F_{1}\right\}\right)$ and $\operatorname{Pl}\left(\left\{F_{1}\right\}\right)=$ $m^{\Omega_{1}}\left(\left\{F_{1}\right\}\right)+m^{\Omega_{1}}\left(\left\{F_{1}, W_{1}\right\}\right)$.

\section{Evaluation of BPAs, beliefs and plausibility functions of} the whole system $S$

First, the vacuous extension is used to extend $m^{\Omega_{1}}$ and $m^{\Omega_{2}}$ to the product space $\Omega_{1} \Omega_{2} \Omega_{S}$. The resulting BPAs are combined using the Dempster combination rule. Then, the resulting BPAs are combined with $m_{\text {Parallel }}^{\Omega_{1} \Omega_{2} \Omega_{S}}$ which represents the relation between the system $S$ and its components 1 and 2. It is given by:

$m_{\text {Parallel }}^{\Omega_{1} \Omega_{2} \Omega_{S}}\left(\left\{\left(W_{1}, W_{2}, W_{S}\right),\left(F_{1}, F_{2}, F_{S}\right),\left(F_{1}, W_{2}, W_{S}\right),\left(W_{1}, F_{2}, W_{S}\right)\right\}\right)=1$

To obtain BPAs of system $S$, the final result is marginalized on $\Omega_{S}$. Belief and plausibility functions are then computed from $m^{\Omega_{S}}$. Formally, the final BPA is defined as follows :

$$
m^{\Omega_{S}}=\left(m^{\Omega_{1} \uparrow \Omega_{1} \Omega_{2} \Omega_{S}} \oplus m^{\Omega_{2} \uparrow \Omega_{1} \Omega_{2} \Omega_{S}} \oplus m_{\text {Config }}^{\Omega_{1} \Omega_{2} \Omega_{S}}\right)^{\downarrow \Omega_{S}}
$$

The system's reliability $R_{S}$ is then given by:

$$
R_{S} \in\left[\operatorname{Bel}\left(\left\{W_{S}\right\}\right), \operatorname{Pl}\left(\left\{W_{S}\right\}\right)\right]
$$

The results of BPAs related to parallel configuration are given in Table I. These results can be generalized to a parallel system of $n$ components with BPAs $m\left(\left\{F_{i}\right\}\right), m\left(\left\{W_{i}\right\}\right)$ and $m\left(\left\{F_{i}, W_{i}\right\}\right)=1-m\left(\left\{F_{i}\right\}\right)-m\left(\left\{W_{i}\right\}\right)$ with $(1 \leq i \leq n)$. In an analogue way, the results for a series system are also shown.

\section{Numerical application: two cases}

- Case I: Aleatory uncertainty Consider a simple parallel system with components 1 and 2. The BPAs of components are given in Table II. Using belief and plausibility measures, the reliability of the system is $R_{s}=0.98$.

When there is no epistemic uncertainty $\left(m^{\Omega_{i}}\left(\left\{F_{i}, W_{i}\right\}\right)=0\right)$, the system's reliability results are identical to the results obtained in the classical probability theory.

- Case II: Aleatory and epistemic uncertainty 
Table I: BPAs and reliability of parallel and series systems with $n$ components

\begin{tabular}{|l|c|}
\hline BPAs & Parallel system \\
\hline$m^{\Omega_{S}}\left\{F_{S}\right\}$ & $\prod_{i=1}^{n} m\left\{F_{i}\right\}$ \\
$m^{\Omega_{S}}\left\{W_{S}\right\}$ & $1-\prod_{i=1}^{n}\left(1-m\left\{W_{i}\right\}\right)$ \\
$m^{\Omega_{S}}\left\{F_{S}, W_{S}\right\}$ & $-\prod_{i=1}^{n} m\left\{F_{i}\right\}+\prod_{i=1}^{n}\left(1-m\left\{W_{i}\right\}\right)$ \\
\hline $\operatorname{Bel}\left\{W_{S}\right\}$ & $1-\prod_{i=1}^{n}\left(1-m\left\{W_{i}\right\}\right)$ \\
$P l\left\{W_{S}\right\}$ & $1-\prod_{i=1}^{n} m\left\{F_{i}\right\}$ \\
\hline \hline BPAs & Series system \\
\hline$m^{\Omega_{S}}\left\{F_{S}\right\}$ & $1-\prod_{i=1}^{n}\left(1-m\left\{F_{n_{i}}\right\}\right)$ \\
$m^{\Omega_{S}}\left\{W_{S}\right\}$ & $\prod_{i=1}^{n} m\left\{W_{n_{i}}\right\}$ \\
$m^{\Omega_{S}}\left\{F_{S}, W_{S}\right\}$ & $\prod_{i=1}^{n}\left(1-m\left\{F_{n_{i}}\right\}\right)-\prod_{i=1}^{n} m\left\{W_{n_{i}}\right\}$ \\
\hline $\operatorname{Bel}\left\{W_{S}\right\}$ & $\prod_{i=1}^{n} m\left\{W_{n_{i}}\right\}$ \\
$P l\left\{W_{S}\right\}$ & $\prod_{i=1}^{n}\left(1-m\left\{F_{n_{i}}\right\}\right)$ \\
\hline
\end{tabular}

Table II: BPAs of components 1 and 2

\begin{tabular}{|c|r|r|r|r|}
\hline & \multicolumn{2}{|c|}{ Case I } & \multicolumn{2}{|c|}{ Case II } \\
\hline Components & $f_{i}$ & $w_{i}$ & $f_{i}$ & $w_{i}$ \\
\hline \hline 1 & 0.1 & 0.9 & 0.3 & 0.65 \\
2 & 0.2 & 0.8 & 0.05 & 0.85 \\
\hline
\end{tabular}

Here, let's consider epistemic uncertainty $\left(m^{\Omega_{i}}\left(\left\{F_{i}, W_{i}\right\}\right)>0\right)$ for components 1 and 2 as shown in Table II. In this case we obtain an interval value for the reliability $R_{s}=[0.9475,0.985]$.

\section{Modeling FaIlure DePEndencies In TBM AND INTERVAL PROBABILITY APPROACHES}

\section{A. Introduction}

Nowadays, complex systems use redundant components in order to increase the overall systems reliability. However, redundant systems are usually subject to multiple failure dependencies [30]. CCFs were the most studied failure dependencies models. Reliability researchers have usually explicitly integrated CCFs in the system's reliability model (FT [9], RBD [31], stochastic Petri nets [8], etc.). Other failure dependencies were integrated implicitly by increasing the failure rates of components [10]. The use of BPAs is proposed to represent failure dependencies and extended operations defined in TBM reliability analysis are used to obtain the whole system's reliability. Implicit and explicit approaches will be presented. The TBM model is compared with an interval-probability model. The values of reliability of components 1 and 2 that would be used from now on, are the same used for case II shown in Table II.

\section{B. The implicit approach}

Let's consider a simple system $S$ composed of two components 1 and 2 in parallel. Reliability experts have mentioned that in $\gamma_{1}$ of system's functioning tests, the failure of component 2 had led to the failure of component 1 . The factor $\gamma_{1}$ is called the dependency factor. The objective is to evaluate the

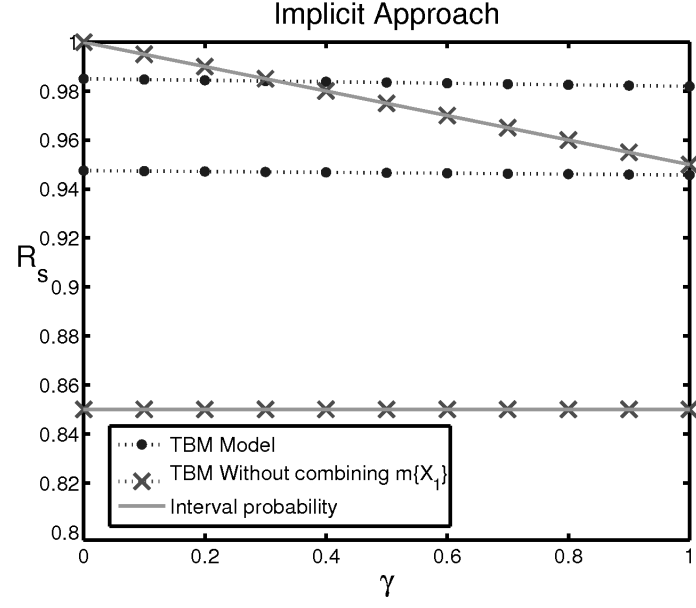

Figure 1: Implicit approach: Results of TBM and Intervalprobability models.

system's reliability $R_{S}$ under these assumptions in both TBM and interval-probability approaches.

1) TBM model: In this approach, the BPAs of components 1 and 2 are given as stated in Eq. 8. The proposed TBM approach is to code the dependence between components 1 and 2 by the conditional BPAs:

$$
\begin{aligned}
& m^{\Omega_{1}}\left[F_{2}\right]\left(\left\{F_{1}\right\}\right)=\gamma_{1} \\
& m^{\Omega_{1}}\left[F_{2}\right]\left(\left\{W_{1}, F_{1}\right\}\right)=1-\gamma_{1}
\end{aligned}
$$

The ballooning extension is used to decondition the BPAs in Eq.10 to $\Omega_{1} \Omega_{2}$. Then, the BPAs obtained are extended to $\Omega_{1} \Omega_{2} \Omega_{S}$ and combined with the BPAs of the simple parallel configuration and the BPAs of components 1 and 2. The final result of the system's reliability after marginalization on $\Omega_{S}$ is given in Eq. 11 and can be observed in Figure 1 as a function of $\gamma_{1}$. The factor $\gamma_{1}$ can be viewed as a correlation factor which assigns an additional BPA to the failure of component 1 knowing failure of component 2 .

$$
\begin{aligned}
\underline{R}_{S} & =\frac{w_{1}+w_{2}-w_{1} w_{2}-\gamma_{1} w_{1} f_{2}}{1-k_{1}\left(f_{2}-f_{2} w_{1}-f_{1} f_{2}\right)} \\
\bar{R}_{S} & =1-\frac{f_{1} f_{2}+\gamma_{1}\left(f_{2}-k_{1}\right.}{1-k_{1}}
\end{aligned}
$$

Where the conflict factor $k_{1}$ is given by: $k_{1}=\gamma_{1} f_{2} w_{1}$.

2) Interval-probability model: The idea of this approach is to use the concepts of interval arithmetic to calculate the reliability. In this case, each probability is represented by an upper and a lower probability noted as $\bar{P}(X)$ and $\underline{P}(X)$ respectively [6]. Then, the probability can be noted as $\bar{P}(X)=[\underline{P}(X), \bar{P}(X)]$. It can be noted that the $\operatorname{Bel}(X)$ and $P l(X)$ corresponding to a BPA can be represented as coherent imprecise probabilities $[\underline{P}(X), \bar{P}(X)]=[\operatorname{Bel}(X), P l(X)]$. Nevertheless, the opposite is not true, as there are some coherent imprecise probabilities that cannot be defined with a corresponding BPA. The corresponding interval-probabilities to the BPAs of components 1 and 2 given in Eq. 12 are: 


$$
\begin{aligned}
& P\left(F_{i}\right)=\left[f_{i}, 1-w_{i}\right] \\
& P\left(W_{i}\right)=\left[w_{i}, 1-f_{i}\right] \quad \text { for } \quad i=1,2
\end{aligned}
$$

The conditional BPAs in Eq. 10 corresponds in the intervalprobability approach to:

$$
P\left(F_{1} / F_{2}\right)=\left[\gamma_{1}, 1\right]
$$

The application of Bayes'rule gives the system's failure probability: $P\left(F_{S}\right)=P\left(F_{1} \cap F_{2}\right)=P\left(F_{1} / F_{2}\right) P\left(F_{2}\right)$. Then, the system's reliability is given in Eq. 12 and shown in Figure 1 as a function of $\gamma_{1}$.

$$
\underline{R}_{S}=w_{2}, \quad \bar{R}_{S}=1-\gamma_{1} f_{2}
$$

3) Discussion: From the Eq. 12, we can see that the interval-probability approach is not sensible to the variation of $f_{1}$ or $w_{1}$. In this case, the reliability is based only on the conditional probability $P\left(F_{1} / F_{2}\right)=\gamma_{1}$ and the values of $f_{2}$ and $w_{2}$. The TBM approach does take into account all the information about the system and its components as it combines all the BPAs stated, but it introduces a conflict factor $k_{1}$ that is caused by the introduction of the conditional BPA (cf. Eq. 10).

To make a similar approach to interval-probability using the TBM, a third approach was analyzed in which the BPA assigned to the component 1 was ignored. In this case, there is no conflict factor because information about component 1 is only taken into account one time with the conditional BPA. The final BPA is obtained by only combining the BPAs assigned for the system configuration, component 2 and dependency factor. Finally, we obtain the same values of reliability as in the interval-probability model (Eq. 12).

It can be concluded that the advantage of the TBM model is that it takes into account the reliability data of component 1 which is not considered in the interval-probability approach due to the use of Baye's rule.

\section{The explicit approach}

In this approach, a virtual component $M$ with two states $E$ and $I$ is considered. This component serves to model dependencies explicitly. The state $E$ of $M$ indicates the presence of CCFs. In this case, the components 1 and 2 are both in failure state $\left(F_{1}, F_{2}\right)$ or in working state $\left(W_{1}, W_{2}\right)$. The state $I$ indicates the absence of CCFs. In this case, components 1 and 2 may have all possible states.

1) TBM model: BPAs of components 1 and 2 are given as stated in Eq. 8. Furthermore, the two previous assumptions are coded by the conditional BPAs:

$$
\begin{gathered}
m^{\Omega_{1} \Omega_{2}}[E]\left(\left\{\left(F_{1}, F_{2}\right),\left(W_{1}, W_{2}\right)\right\}\right)=1 \\
m^{\Omega_{1} \Omega_{2}}[I]\left(\left\{\left(F_{1}, F_{2}\right),\left(F_{1}, W_{2}\right),\left(W_{1}, W_{2}\right),\left(W_{1}, F_{2}\right)\right\}\right)=1
\end{gathered}
$$

The frame of discernment of $M$ is then given by: $\Omega_{M}=$ $\{E, I\}$ and the BPAs related to $M$ are given in Eq. 15 .

$$
\begin{array}{ll}
m^{\Omega_{M}}(\{E\})=\delta_{1}, & m^{\Omega_{M}}(\{I\})=\delta_{2} \\
m^{\Omega_{M}}(\{E, I\})=1-\delta_{1}-\delta_{2} &
\end{array}
$$

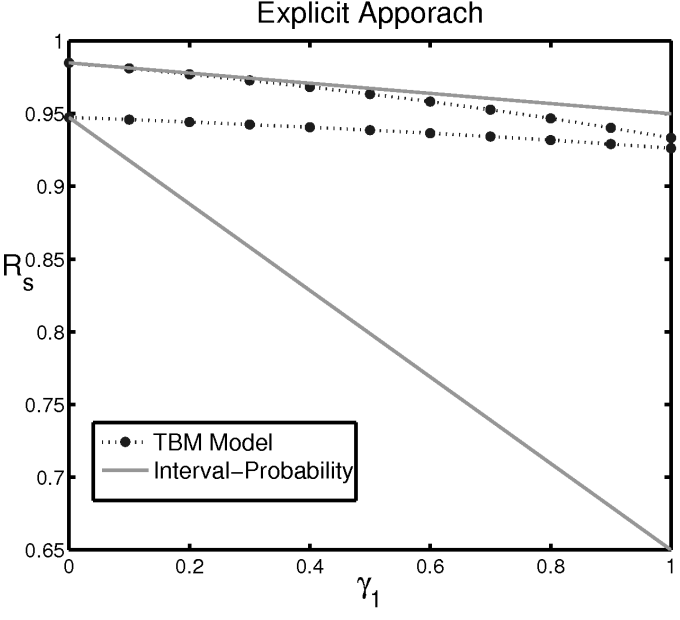

Figure 2: Explicit approach: Results of TBM and Intervalprobability models.

The factor $\delta_{1}$ assigns an additional BPA to the fact that components 1 and 2 are either working or have failed when the virtual component $M$ is in the state $E$. The factor $\delta_{2}$ assigns a mass value to the fact that the components 1 and 2 may have all possible states.

The BPAs of Eq.13 and Eq.14 are deconditioned to $\Omega_{1} \Omega_{2} \Omega_{M}$. Then, the BPAs obtained and the BPAs of Eq. 15 are extended to $\Omega_{1} \Omega_{2} \Omega_{S} \Omega_{M}$. The obtained BPAs are then combined with the BPAs of the parallel structure. The final results of the system's reliability after marginalization on $\Omega_{S}$ are given in Eq. 16 and can be seen in Figure 2 as a function of $\delta_{1}$. Note that $\delta_{2}$ doesn't appear in the final solution, which means that the epistemic uncertainty of the state of the machine $M$ doesn't have any influence when the TBM model is used. In this case, the BPA of Eq. 13 also introduces a conflict factor $\left.k_{2}=\delta_{1}\left(f_{1} w_{2}+f_{2} w_{1}\right)\right)$ due to the fact that for the state $E$, the events $\left(F_{1}, W_{2}\right)$ and $\left(W_{1}, F_{2}\right)$ can't happen. Thanks to this, when the BPAs are combined, there is a conflict between the BPA's components and the conditional BPA.

$$
\begin{aligned}
\underline{R}_{S} & =\frac{w_{1}+w_{2}-w_{1} w_{2}-\delta_{1}\left(f_{1} w_{2}+f_{2} w_{1}\right)}{1-k_{2}} \\
\bar{R}_{S} & =1-\frac{f_{1} f_{2}+\delta_{1}\left(f_{1}+f_{2}-f_{1} w_{2}-f_{2} w_{1}-2 f_{1} f_{2}\right)}{1-k_{2}}
\end{aligned}
$$

2) Interval-probability model: In this case, the values of $P\left(F_{i}\right)$ and $P\left(W_{i}\right)$ are the same used for the implicit approach. As $\delta_{1}$ and $\delta_{2}$ are variable and it is not known which one is greater than the other for a given combination of values, the interval values of $P(E)$ and $P(I)$ are expressed using the min and max functions as follows:

$$
\begin{aligned}
& P(E)=\left[\min \left(\delta_{1}, 1-\delta_{2}\right), \max \left(\delta_{1}, 1-\delta_{2}\right)\right] \\
& P(I)=\left[\min \left(\delta_{2}, 1-\delta_{1}\right), \max \left(\delta_{2}, 1-\delta_{1}\right)\right]
\end{aligned}
$$

Also note that $\delta_{1}+\delta_{2} \leqslant 1$. For the conditional probability $P\left(F_{1} \cap F_{2} / E\right)$, the largest possible interval is used so that every possible value is taken into account. 


$$
\begin{aligned}
& P\left(F_{1} \cap F_{2} / E\right)=\left[\min \left(f_{1}, f_{2}\right), \max \left(1-w_{1}, 1-w_{2}\right)\right] \\
& P\left(F_{1} \cap F_{2} / I\right)=P\left(F_{1}\right) P\left(F_{2}\right)
\end{aligned}
$$

In this case, the total probability theorem is used to calculate $P\left(F_{s}\right)$ :

$$
\begin{aligned}
& P\left(F_{s}\right)=P\left(F_{1} \cap F_{2}\right) \\
& P\left(F_{s}\right)=P\left(F_{1} \cap F_{2} / E\right) P(E)+P\left(F_{1} \cap F_{2} / I\right) P(I)
\end{aligned}
$$

Finally, knowing that $R_{s}=1-P\left(F_{s}\right)$, the system's reliability is given in Eq. 17 and shown in Figure 2 as a function of $\delta_{1}$.

$$
\begin{array}{r}
\underline{R}_{S}=1-\max \left(1-w_{1}, 1-w_{2}\right) \max \left(\delta_{1}, 1-\delta_{2}\right)- \\
-\left(1-w_{1}\right)\left(1-w_{2}\right) \max \left(\delta_{2}, 1-\delta_{1}\right) \\
\bar{R}_{S}=1-\min \left(f_{1}, f_{2}\right) \min \left(\delta_{1}, 1-\delta_{2}\right)- \\
-f_{1} f_{2} \min \left(\delta_{2}, 1-\delta_{1}\right)
\end{array}
$$

3) Discussion: For the case analyzed, it can be noted that the TBM model gives a more precise interval for the system's reliability. On the other hand, the interval-probability model becomes much more imprecise as $\delta_{1}$ grows. It can also be seen that the results obtained with the TBM are included in the results of the interval-probability model.

\section{CONCLUSIONS AND FUTURE WORK}

The TBM theory has recently attracted the attention of reliability engineering community. This paper proposes a TBM based model and compares it with an interval-probability based model. It takes into account failure dependencies in reliability evaluations under both epistemic and aleatory uncertainties. The proposed TBM reliability model was applied to evaluate the reliability of a parallel system with two components and the dependencies were implicitly and explicitly modeled. As we can see from the results, with the TBM approach, the epistemic uncertainties and the dependencies present in our systems can be modeled. As it combines all of the BPAs to obtain the results, it takes into account all of the information known for the system. Future work would be focused on other ways of coding the dependencies hypothesis and different methods for incorporating them in the evaluation or reliability of complex systems.

\section{REFERENCES}

[1] K. Durga Raoa, H. Kushwahaa, A. Vermab, and A. Srividyab, "Quantification of epistemic and aleatory uncertainties in level-1 probabilistic safety assessment studies," Reliability Engineering and System Safety, vol. 92, pp. 947-956, 2007.

[2] E. Zio, "Reliability engineering: Old problems and new challenges," Reliability Engineering and System Safety, vol. 94, pp. 125-141, 2009.

[3] R. L. Winkler, "Uncertainty in probabilistic risk assessment," Reliability Engineering and System Safety, vol. 54, 1996.

[4] W. L. Oberkampf, J. C. Helton, C. A. Joslyn, S. F. Wojtkiewicz, and S. Ferson, "Challenge problems: uncertainty in system response given uncertain parameters," Reliability Engineering and System Safety, vol. 85, pp. 11-19, 2004.

[5] R. Flage, T. Aven, and E. Zio, "Alternative representations of uncertainty in system reliability and risk analysis-review and discussion," European Safety and Reliability Conference (ESREL), Valencia, SPAIN, vol. 43, pp. 22-25, 2008.
[6] P. Walley, Statistical reasoning with imprecise probabilities. New York : Chapman and Hall, 1991.

[7] P. Smets, "Belief functions versus probability functions," in Uncertainty and Intelligent Systems, 1988, pp. 17-24.

[8] R. M. Fricks and K. S. Trivedi, "Modeling failure dependencies in reliability analysis using stochastic petri nets," Proceedings of European Simulation Multiconference (ESM 97), Istanbul, Turkey, 1997.

[9] J. Vaurio, "Common cause failure probabilities in standby safety system fault tree analysis with testing-scheme and timing dependencies," Reliability Engineering and System Safety, vol. 79, pp. 43-57, 2003.

[10] Misra and B. Krichna, Handbook of Performability Engineering. Springer Verlag, 2008.

[11] A. P. Dempster and A. Kong, "Uncertain evidence and artificial analysis," Journal of Statistical Planing and Inference, vol. 20, pp. 355-368, 1988.

[12] M. Guth, "A probabilistic foundation for vagueness and imprecision infault-tree analysis," IEEE Transactions on Reliability, vol. 40, pp. $563-$ $571,1991$.

[13] W. C. Chin and V. Ramachandran, "Evidence sets approach for web service fault diagnosis," Malaysian Journal of Computer Science, vol. 13, pp. 84-89, 2000.

[14] I. Kozine and Y. Filimonov, "Imprecise reliabilities: experiences and advances," Reliability Engineering and System Safety, vol. 67, pp. 75$83,2000$.

[15] R. G. Almond, Graphical belief modeling. CRC Press, 1995.

[16] U. K. Rakowsky and U. Gocht, "Reasoning in reliability-centred maintenance based on a dempster-shafer approach," Proceedings of the Institution of Mechanical Engineers, Part O: Journal of Risk and Reliability, vol. 222, pp. 605-612, 2008.

[17] S. Pashazadeh and M. Sharifi, "Reliability assessment under uncertainty using dempster-shafer and vague set theories," Computational Intelligence for Measurement Systems and Applications, CIMSA 2008, vol. 1, pp. 131-136, 2008.

[18] C. Simon and P. Weber, "Evidential networks for reliability analysis and performance evaluation of systems with imprecise knowledge," IEEE Transactions on Reliability, vol. 58, pp. 69-87, 2009.

[19] P. Smets and R. Kennes, "The transferable belief model," Artificial Intelligence, vol. 66, pp. 191-243, 1994.

[20] A. P. Dempster, "Upper and lower probabilities induced by multivalued mapping," Annals of Mathematical Statistics, vol. 38, pp. 325-339, 1967.

[21] G. Shafer, A Mathematical Theory of Evidence. Princeton University Press, 1976.

[22] S. Fabre, A. Appriou, and X. Briottet, "Presentation and description of two classification methods using data fusion based on sensor management," Information Fusion, vol. 2, pp. 49-71, 2001.

[23] D. Mercier, G. Cron, T. Denoeux, and M. Masson, "Fusion of multilevel decision systems using the transferable belief model," Proceedings of FUSION 2005, Philadelphia, 2005.

[24] T. Denoeux, "A k-nearest neighbor classification rule based on Dempster-Shafer theory," IEEE Transactions on Systems Man and Cybernetics, vol. 25, no. 5, pp. 804-813, 1995.

[25] D. Mercier, G. Cron, T. Denoeux, and M. H. Masson, "Decision fusion for postal address recognition using belief functions," Expert Systems with Applications, vol. 36, no. 3P1, pp. 5643-5653, 2009.

[26] P. Smets, "The application of the transferable belief model to diagnosis problems," International Journal of Intelligent Systems, vol. 13, pp. 127158, 1998.

[27] K. Sentz and S. Ferson, Combination of evidence in Dempster-Shafer theory. Sandia National Laboratories, 2002.

[28] P. Smets, "The combination of evidence in the transferable belief model," IEEE Transactions on Pattern Analysis and Machine Intelligence, vol. 12, pp. 447-458, 1990.

[29] G. Shafer, P. P. Shenoy, and K. Mellouli, "Propagating belief functions in qualitative markov trees," International Journal of Approximate Reasoning, vol. 1, pp. 349-400, 1987.

[30] Y. Haiyang, C. Chengbin, E. Chatelet, and F. Yalaoui, "Reliability optimization of a redundant system with failure dependencies," Reliability Engineering and System Safety, vol. 92, pp. 1627-1634, 2007.

[31] S. Distefano and A. Puliafito, "Dynamic reliability block diagrams vs dynamic fault trees," Reliability and Maintainability Symposium, Orlondo, USA, 2007. 